\title{
An Efficient Multi-Beam Array Architecture for L-Band Secondary Surveillance Radars
}

\author{
Hadi ALIAKBARIAN ${ }^{1}$, Mohammad KHAK ${ }^{2}$, Morteza SHAHPARI ${ }^{3}$, Ali NOORAEI YEGANEH ${ }^{1}$, \\ Farhad MAZLUMI ${ }^{4}$, Seyed Hesamoddin NAJMOLHODA ${ }^{2}$, Reza BAGHLANI ${ }^{2}$, Rasoul SALIMI NEJAD ${ }^{2}$ \\ ${ }^{1}$ Electrical Engineering Faculty, KN Toosi University of Technology, Tehran, Iran \\ ${ }^{2}$ Basamad Azma Co., Tehran, Iran \\ ${ }^{3}$ School of Engineering, Griffith University, Brisbane, Australia \\ ${ }^{4}$ Civil Aviation Technology College, MehrAbad International Airport, Tehran, Iran
}

h.aliakbarian@ieee.org

Submitted June 18, 2018 / Accepted December 30, 2018

\begin{abstract}
In this paper, the design and fabrication of a large array antenna with three required, Sum, Difference and Control beams for a monopulse Secondary Surveillance Radar (SSR) is presented. A special array element, which is a high gain dual dipole structure, is designed and tested. This element has 9.6 dB gain in $1060 \mathrm{MHz}$ and does not need any balun. The main challenge of extracting three beams out of one beam feeding networks (BFN) has been resolved efficiently by maximum integration of all three in one network, reducing the number of required modules to one half. The complete 33-element array, working in $1030 \mathrm{MHz}$ and $1090 \mathrm{MHz}$ is designed based on the Taylor array factor. The complex feed network of this 6-meter long array has successfully been synthesized as modular as possible. Sum pattern was designed for $2.7^{\circ}$ azimuth beam width and $-20 \mathrm{~dB}$ side lobe level and Diff pattern with a deep null in the boresight of the Sum pattern. The Ctrl beam was also designed in order to cover the Sum beam except in the direction of the main lobe.
\end{abstract}

\section{Keywords}

Secondary Surveillance Radar, beam forming network, monopulse array, Taylor distribution

\section{Introduction}

Secondary Surveillance Radars (SSR) is an essential part of Aviation Traffic Control (ATC) systems. The role of this radar is to track and identify any aircraft in the area in addition to its place and altitude. One of the biggest advantages of these radars is their long range and lower power consumption which are made possible by using a transponder in all aircraft to respond to interrogations from the ground systems [1]. Furthermore, clutter effect is negligible in these types of radars.

Monopulse array systems are one of the most favorite structures used in SSR systems [4]. The monopulse anten- nas are available in planar array type [5, 6, 7], modal Horn Type [8], [9], reflector type [10-12] or even waveguide arrays [13]. Use of monopulse methods [10], [4] for ranging and angle of the arrival estimations which necessitate generation of two SUM and Difference (DIFF), and for more reliability in SSR, use of Control (CTRL) beams out of its antenna system. In the SUM beam, there should be a sharp high gain main beam beside very low levels of side lobes. The DIFF pattern needs to have a deep null at the direction of SUM's maximum. The CTRL beam only needs to cover SUM's side lobes while being below the SUM in the main beam [2]. Many of the above mentioned solutions such as [6], [7], and [13] cannot support the CTRL beam.

The interrogating signal, which aims at identifying the passing aircraft, is received by the aircraft from the rotating ATC system's two beams sequentially, first from SUM and then from CTRL. If the aircraft receives higher signal level from the latter than that of the former, it means that it is not in the main beam and thus ignores it. However, if the SUM's level is higher than CTRL, it replies accordingly to ATC. This signal is received by the ATC's SUM and DIFF pattern in the main beam direction. The received level in SUM is higher than that of DIFF (which is ideally zero on the direction of DIFF's null). The relation between these two values, namely SUM/DIFF, is inversely related to the precise direction of the aircraft seen from the ground station. Also by comparing the received relative phase of SUM/DIFF we are able to determine whether the object is at the right or left side of the beam center [1], [2]. Based on International Civil Aviation Organization (ICAO) standards, the interrogation frequency is $(1030 \pm 0.01) \mathrm{MHz}$ and the reply frequency is $(1090 \pm 1) \mathrm{MHz}$ [3].

One of the main challenges of an SSR array design is the design of its antenna which requires three different beams out one antenna. It is not recommended to design three different antennas due to the dependency of all three patterns in their processing part and also large amount of required weight and space. On the contrary, it is recommended to generate all three patterns by manipulating the 
array's excitation coefficients [15] or type of mode excitation [8]. For CTRL pattern only, one good suggestion is the use of a simple monopole antenna or even one of the array elements. The modal horn type does not have enough gain and cannot generate efficient fan beam. Reflector type monopulse antennas are also very unsuitable choices for utilizing in L-band SSR. Thus the array type monopulse antenna is chosen for implementation.

The element factor in array design has a major role on final properties of the array antenna such as power handling, size, and radiation patterns. Monopoles and dipoles are usual elements chosen for antenna base element. Dipole element is a good choice because of its stable radiation pattern in frequency domain, good beam shape, enough bandwidth, and ability to become high-gain [16].

Another important matter in SSR arrays is the design of feed network. It is extremely inefficient, inaccurate and unnecessary to design three antenna arrays, each one with its own BFN, separately. A solution is to design three BFNs separately and then to combine/split them right at the input of antenna array elements. A well-known more-efficient alternative solution is to design a multi-beam feeding network. This idea, which has been used in radar systems for a long time $[15,17,18]$, paves the way for maximum integration among all three beams, SUM, DIFF, and CTRL, resulting in miniaturization, simplification and reducing the price of the system.

In order to generate SUM and DIFF patterns only, custom designed power splitter/combiner, and 180-degree hybrids are needed. A suitable feed network is proposed in [19] for SUM and DIFF pattern, but it is not able to generate CTRL pattern. Moreover, it is not optimized choice for DIFF pattern. Other options are proposed in [13] and [20] for SSR arrays. The details of our proposed BFN are presented in Sec. 3.
In this paper, a 33-element array design for all three SUM, DIFF and CTRL beam is proposed. The designed array is very high power, and each of the array elements is able to tolerate above $2 \mathrm{~kW}$ of pulsed power. The primary design considers better than $-20 \mathrm{~dB}$ of side lobe level which has been obtained by Taylor distribution. In Sec. 2, the antenna element design is discussed. In Sec. 3 and 4, the design of array and its feed network components are discussed. The system fabrication is presented in Sec. 5 and finally, the paper is concluded in Sec. 6 .

\section{Antenna Element Design}

Antenna arrays used in SSRs normally use one-dimensional linear arrays due to the fact that they need to shape the beam primarily in the direction of horizon compared to more complicated two-dimensional planar arrays. The required elevation beamwidth in such antennas is not normally more than 40 degrees. A vertical dipole element has an elevation beamwidth of around 80 degrees as expected. Therefore there is still room to sharpen its elevation radiation pattern and consequently increase antenna's gain. However, a linear array narrows its beam only in the direction of its axis while does not affect the radiation pattern in the plane orthogonal to it.

To achieve higher gain and have narrow beamwidth in the elevation plane, a dual dipole element has been used. The proposed dual dipole element, which is shown in Fig. 1, is composed of two dipoles, half wavelength apart and are placed quarter wavelength from a ground plane. The ground plane converts the high-gain omnidirectional pattern of the two-dipole sub-array into directional beam which is preferred for SSR array. As the feeding line is symmetrical, this dipole element does not need any balun. In the other words, the balun is combined with the trans-

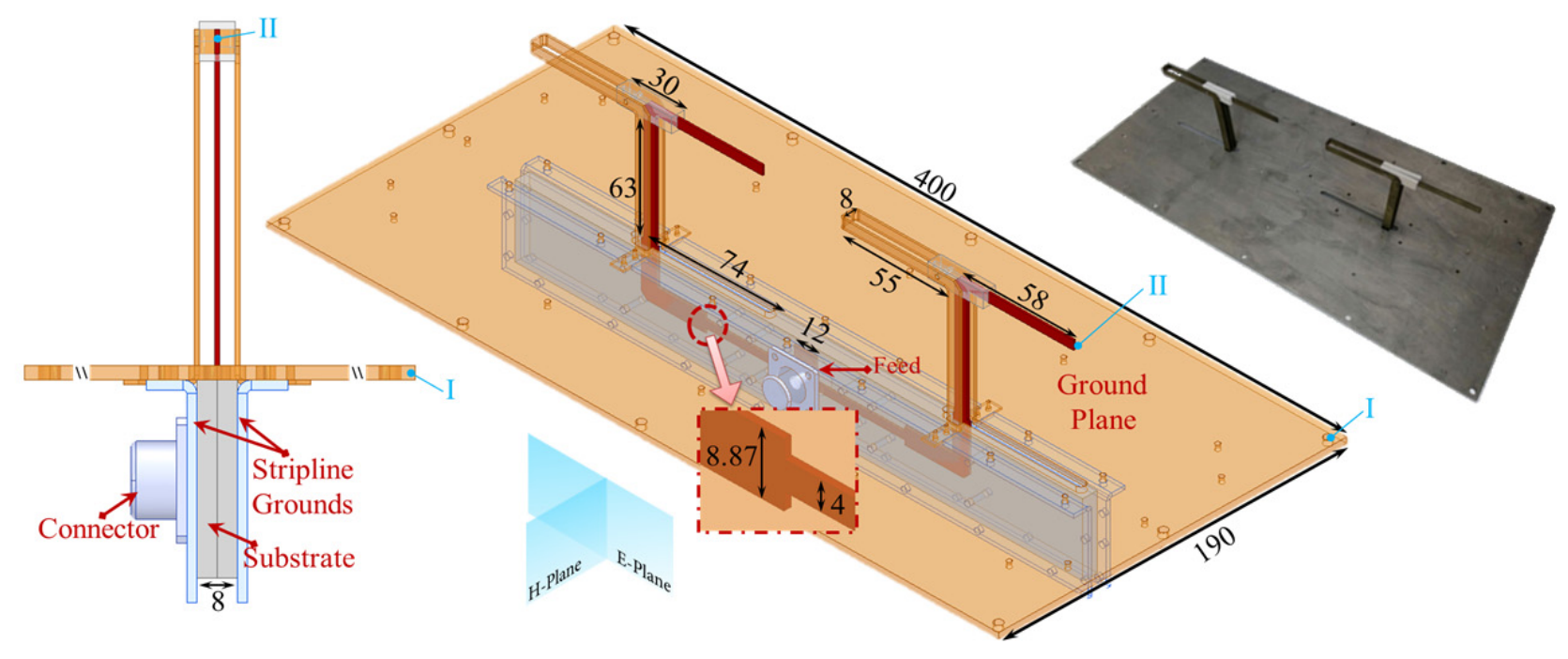

(a) (b) (c)

Fig. 1. (a) Left view. (b) Perspective view of the antenna element. (c) Fabricated antenna element (dimensions are in millimeters). 
mission line which is a stripline sandwiched between grounded walls. For more compaction, a T-junction splitter made of stripline feeds splits power into both dipoles as shown in Fig. 1(a) and (b). Two quarter wavelength impedance converters with $95 \Omega$ characteristic impedance match both dipoles' input impedance to $50 \Omega$. In Fig. 2, the result of $S_{11}$ for this antenna is presented illustrating a good agreement between the simulated $S_{11}$ and its measurement. The antenna return loss is below $-25 \mathrm{~dB}$ within the target $1030 \mathrm{MHz}$ to $1090 \mathrm{MHz}$ band. The current distribution of the dipole arms, depicted in Fig. 3 for $1030 \mathrm{MHz}$, shows that the currents on both arms are in the same direction and also has electrically quarter wavelength length. The symmetrical excitation of the dipoles along with the observation of regular current on the arms guarantee a proper radiation pattern for the element and introduce it as a good candidate for other applications as well. The transmission line also has a very low contribution to the pattern. These are confirmed in Fig. 4 where the simulated and measured radiation patterns in $1030 \mathrm{MHz}$ and $1090 \mathrm{MHz}$ are shown and compared with very good agreement.

\section{Array Design}

The proposed three-beam feeding network is designed in a way that minimizes the size of the array and removes the unnecessary modules from the network. Therefore, it is tried to reuse as maximum feed components as possible for the generation of DIFF and SUM beams and finally a sample of SUM beam is used to generate a good CTRL beam. This results in reducing the number of required modules to less than one half compared to the case which all three beams are generated separately. According to Fig. 4(a) the dipole array's gain is around $8.8 \mathrm{~dB}$ and $9.2 \mathrm{~dB}$, at $1030 \mathrm{MHz}$ and $1090 \mathrm{MHz}$, respectively.

In order to miniaturize the final BFN design, DIFF and CTRL array coefficients are obtained from the initially designed coefficients of SUM pattern as the reference beam. The assumed fan beam gain of $24 \mathrm{~dB}$ is equivalent to azimuth beam width of $2.7^{\circ}$ and elevation beam width of $39^{\circ}$. Having an element gain of $9 \mathrm{~dB}$, the remaining $15 \mathrm{~dB}$ is realizable by a 32-element uniformly excited linear array. The numbers of elements are set to be an odd number $(29,31$ or 33$)$ in order to optimize feed network structure. As there is no electrical beam scanning the structure excitation values are symmetric. Therefore the feed network structure is divided into five sub-array parts I, II, III, IV, and V, shown in Fig. 5(a), which are responsible for different parts of three beams.

Sidelobe level is very important in SSR monopulse radars which is normally designed to be around $24 \mathrm{~dB}$ or more while the SLL of a uniform distribution is only $13 \mathrm{~dB}$. Among all low side lobe distributions, such as binomial or Chebyshev, Taylor distribution is the optimum since it has the lowest SLL while minimally sacrifices beam-

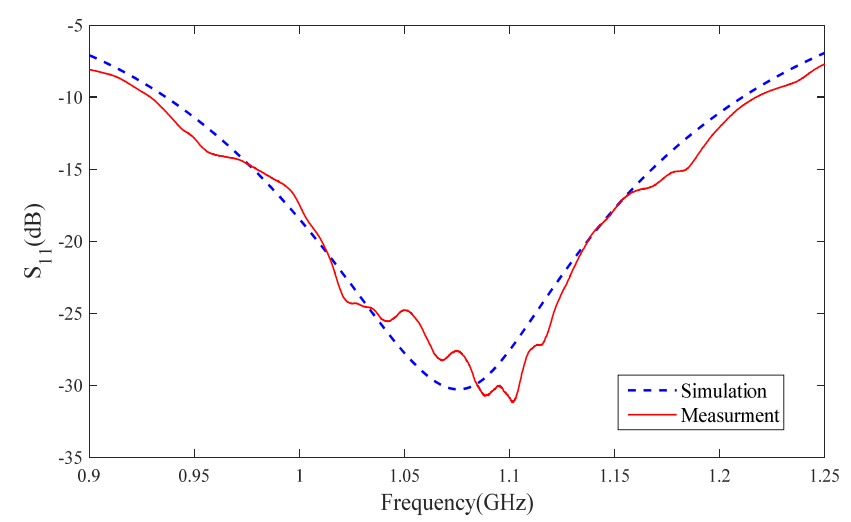

Fig. 2. Simulated vs. measured $\left|S_{11}\right|$.

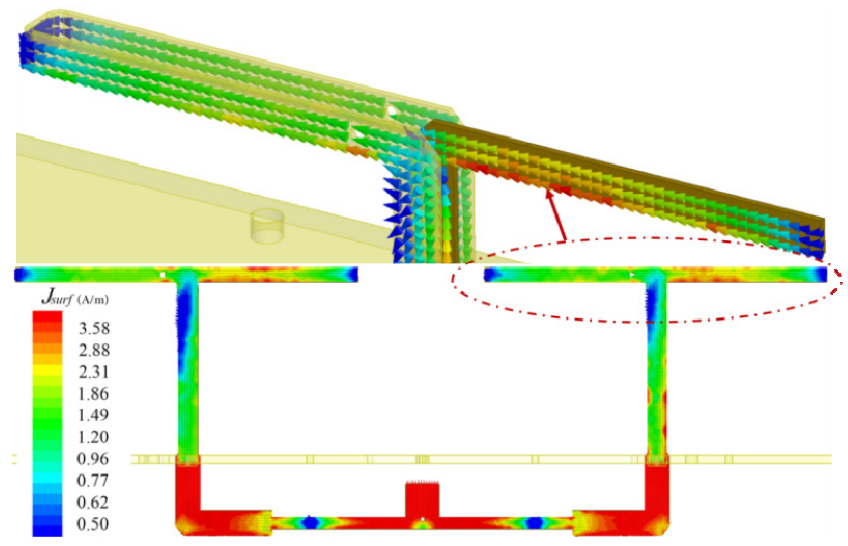

Fig. 3. Current distribution on dipole antenna in $1030 \mathrm{MHz}$.

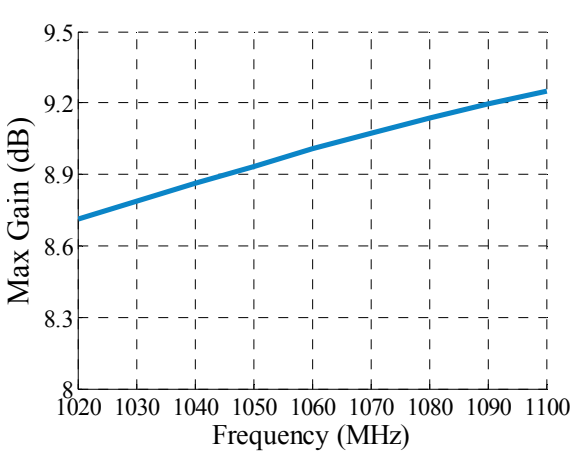

(a)

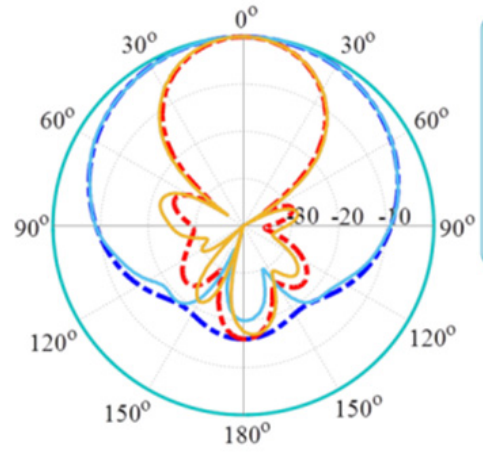

(b)
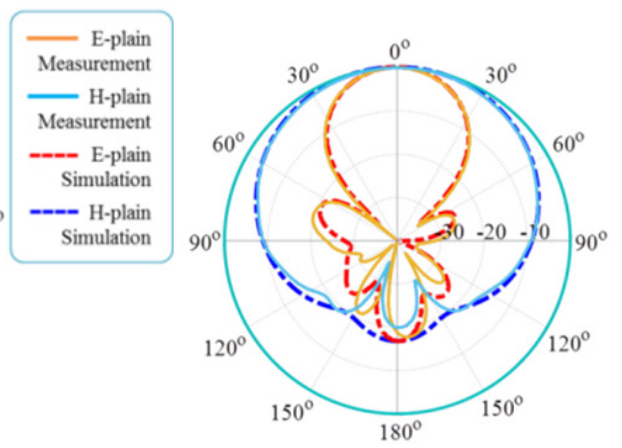

(c)

Fig. 4. Simulated (a) max gain, (b) antenna element radiation pattern in $1030 \mathrm{MHz}$ and (c) $1090 \mathrm{MHz}$ 


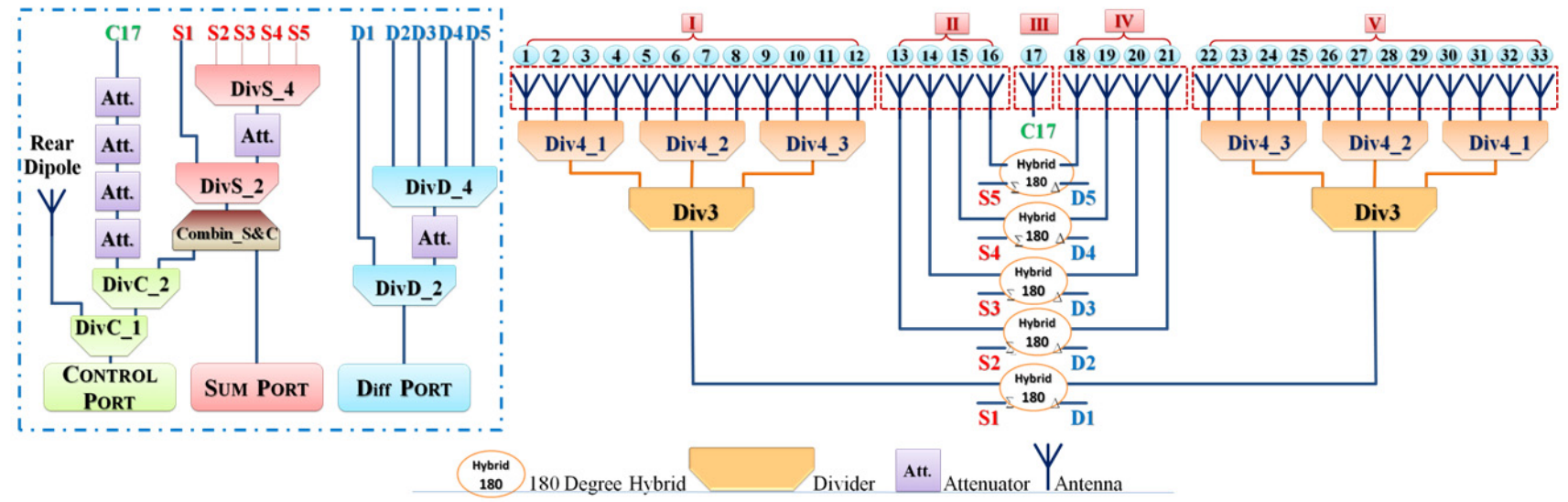

(a)

(b)

Fig. 5. Five sections of SSR feed network in two parts. (a) Parts near output ports (b) parts near antenna elements.

width and gain. Taylor distribution [21] reduces the level of the greatest side lobes by placing $N$ nulls on the first $N$ maximums of the uniform distribution array factor. The resulted array factor is

$$
\delta(u)=\frac{\sin \pi u}{\pi u} \frac{\prod_{n=1}^{\bar{n}-1}\left(1-u^{2} / u_{n}^{2}\right)}{\prod_{n=1}^{\bar{n}-1}\left(1-u^{2} / n^{2}\right)}
$$

with auxiliary variables $u$ and $u_{n}$ are obtained from

$$
\begin{gathered}
u=\frac{2 a}{\lambda}\left(\cos \theta-\frac{\beta}{k}\right), \\
u_{n}=\bar{n}\left[\frac{a^{2}+(n-1 / 2)^{2}}{a^{2}+(\bar{n}-1 / 2)^{2}}\right]^{\frac{1}{2}}
\end{gathered}
$$

where $n$ is the number of elements and $a$ is the distance between them, in our case $19 \mathrm{~cm} . \beta$ is the propagation constant and $\lambda$ the wavelength.

For the DIFF pattern, a deep null is required in the center of the pattern. Therefore, the array factor needs to have equal amplitude coefficients on both left and right sides of the array but with $180^{\circ}$ phase shift. This is done by getting SUM beam's values and applying the phase shift of $180^{\circ}$ on one side of its network (part I and II versus part IV and V) while ignoring center element (part III). Unfortunately, these values increase the SLL of the DIFF beam. Thus, the optimization of DIFF beam is done by changing the weight of part II and IV, compared to part I and V.

A good solution for CTRL beam's array factor is an omnidirectional array factor with a null in the center direction. To achieve that, a $-14 \mathrm{~dB}$ sample of SUM beam is subtracted from the center element of the array $(1-0.177 u(\theta))$, which is seen in Fig. 5(a), component DivC_2. Here, a direction signal from $\mathrm{C} 17$ is subtracted from a sample of SUM beam extracted from Combin_S\&C. Due to very low side lobe of SUM beam, the resulted array factor is approximately equal to antenna elements beam in all directions except in the center direction where SUM beam and center element are equal in amplitude and $180^{\circ}$ different in phase resulting in a null in that direction. Therefore the final CTRL beam is above SUM beam's side lobe by $10 \mathrm{~dB}$. Coefficients of arrays are illustrated in Fig. 6.

The final full wave simulated radiation pattern of all three beams including the element factor is presented in Fig. 7. As can be seen, the SUM pattern has a $24 \mathrm{~dB}$ gain and a SLL of $25 \mathrm{~dB}$. Also, the CTRL pattern is always above SUM pattern except the desired front direction and undesired back lobe radiation. This problem can be improved by adding a rear antenna, exactly similar to front dipole, at the back as shown in Fig. 5(b). Adding this element at the back creates multiple nulls around $-90^{\circ}$ and $90^{\circ}$. Another method to resolve this problem is to use signal processing techniques which is the case in our system.

\section{Feed Network}

Three separate ports CTRL, DIFF and SUM are seen out of a SSR array in Fig. 5(b). When tracking an aircraft, SUM and DIFF patterns are simultaneously needed. But at the time of interrogation, SUM and CTRL beams are sequentially required. All divider modules have been designed with the same phase transfer characteristics to achieve identical phase distribution over the array. Based on the feed network diagram, shown in Fig. 5, the network can be divided into two main parts, the part near the output ports in Fig. 5(a) and the part near the antenna elements in Fig. 5(b). S1 to S5, D1 to D5 and C17 are the connections between the two, which come from the antenna elements and go to all three ports. The CTRL beam coefficients are the constant coefficient of SUM beam except for the center elements. Illustrated in the feed network (Fig. 5), the rear dipole is separated first by DivC_1. Then, the C17 (center element) is separated by using DivC_2. Finally, current path to other elements is provided by Combin_S\&C power splitter /combiner as a sample of SUM beam. It is seen here that S1 to S5 are shared paths for both SUM and CTRL beams and D1 to D5 are paths for DIFF beam. 
Shown in Fig. 5(b), S1 and D1 paths are specified for $\mathrm{I}$ and V sub-arrays while S2 to S5 and D2 to D5 paths are assigned to sub-arrays II and IV. Based on the coefficients in Fig. 6, the ideal coefficient for center element is +1 for SUM beam, -1 for CTRL beam, and 0 for DIFF beam. However for simplicity of implementation, it is assumed 0 for the SUM beam, as can be seen in Fig. 5, and C17 only feeds the CTRL beam.

The use of $180^{\circ}$ hybrid is necessary to have summation $\left(\sum\right.$ port) and subtraction ( $\Delta$ port). Summation is needed for SUM and CTRL beam while subtraction of elements of opposite side of the array is needed for DIFF pattern by using $180^{\circ}$ hybrid. The design of each splitter/combiners is different based on power distribution which is needed for each antenna element. As shown in Fig. 5(b), there are three different 1-to-4 power splitters, namely Div4_3, Div4_2 and Div4_1 and also one 1-to-3 splitter, Div 3 , in sub-array I. The role of Div3 is to split/combine in/out signals of all three 1-to-4 splitters, Div4_1, Div4_2, and Div4_3. The unequal power splitters are designed based on relative distribution of currents on elements. For instance, for Div4_1, it is $0.357,0.371$, 0.398 , and 0.436 , respectively. These values are scaled in CTRL pattern by $-14 \mathrm{~dB}$ because of $\mathrm{DivC}_{2} 2$ and Combin_S\&C, shown in Fig. 5(a). Based on these values, the impedance values of a simple 1-to-4 T-junction splitter can be easy calculated with basic microwave references [22]. The design procedures for Div4_3, Div4_2 and also Div3 are the same. The exact same components are made for the other half of the network in sub-array V.

S2, S3, S4, and S5 which are summed signals of subarray II and IV are splitted/combined by applying their coefficients in DivS_4, one attenuator and DivS_2. The same procedure applies the array coefficients of DIFF beam by using DivD_4, DivD_2 and another attenuator in between.

\section{Component Design}

All required feed network components have been designed and fabricated with the simplest and most efficient configuration. They include power combiners/splitters, couplers, attenuator, and needless to say, the hidden components of the network, phase shifting coaxial cables. These cables with various phase shift values are needed to compensate for the difference between the expected phase difference at each place of the network and the real measured one.

The phase shift of all 64 coaxial cables connecting all components (26 feed network modules plus antenna elements) is accurately calculated in a way not to affect the phase distribution of the modules. At the same time, the length of each cable should be enough to connect corresponding modules, most of which are shown in Fig. 8 in the feed network's 6-meter frame. Afterwards the lengths of the cables are adjusted in order to guarantee equal path phase shifts, taking the connecting N-type connector into account as well. These lengths start from $85 \mathrm{~mm}$ up to $1148 \mathrm{~mm}$.

For the microstrip power dividers, the well-known, simple, and efficient method of arbitrary power division ratio is used based on the difference between the widths of output microstrip ports and therefore their characteristic impedance [19], [22]. These characteristic impedances for an $N$-way power divider $\left(Z_{i}\right)$ are given by simultaneous solution of $N$ equations, namely (4) and ( $N-1)$ equations (5)

$$
\begin{gathered}
\frac{1}{Z_{0}}=\sum_{i=1}^{N} \frac{1}{Z_{i}}, \\
\frac{P_{i}}{P_{i+1}}=\frac{Z_{i+1}}{Z_{i}}
\end{gathered}
$$

where $Z_{0}$ is the input port impedance, $50 \Omega, P_{i}$ are the expected relative output power of the $i$-th port.

As the layouts of three of the components are shown in Fig. 9, all of them are designed to be matched from the input ports. Also, the electrical length of all lines is equal as can be seen in Fig. 9. The impedance of all output ports are changed to $50 \Omega$ at the ports.

For the hybrid couplers, a conventional rat-race configuration [19], [22] shown in Fig. 10 satisfies the requirements. For the $-15 \mathrm{~dB}$ coupler, used to sample SUM signal

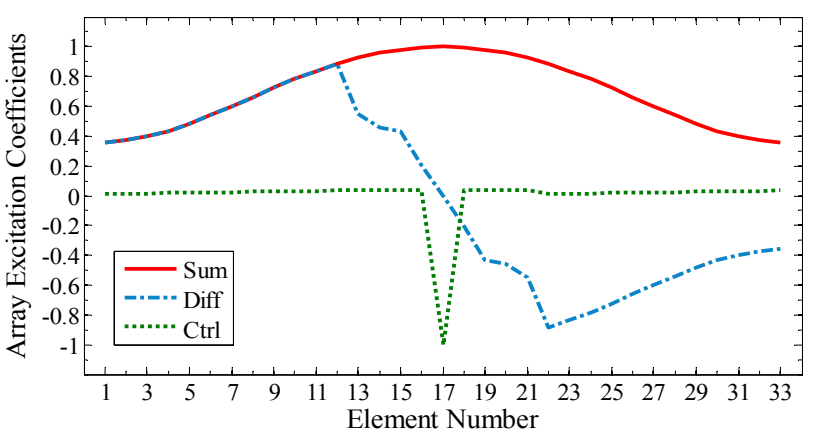

Fig. 6. Array excitation coefficients of all three beams (amplitude and phase).

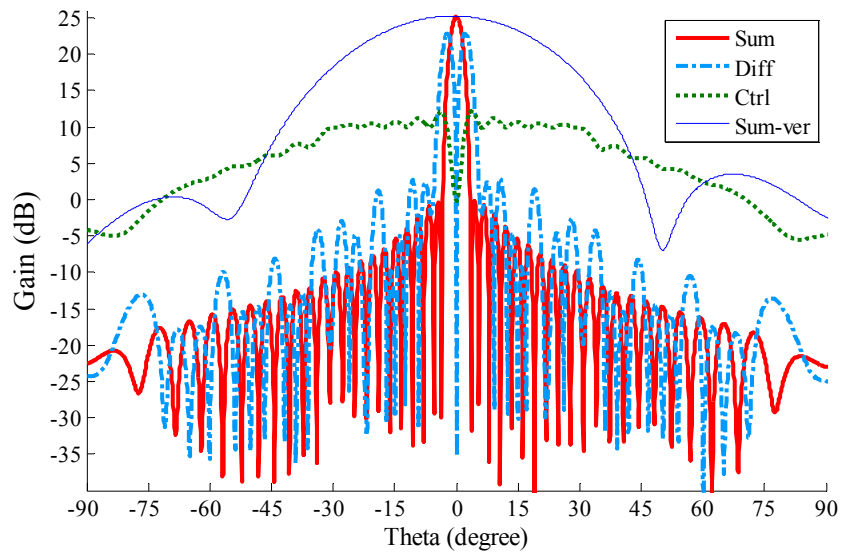

Fig. 7. Simulated radiation pattern of SUM, DIFF, CTRL and SUM in vertical in $1030 \mathrm{MHz}$. 


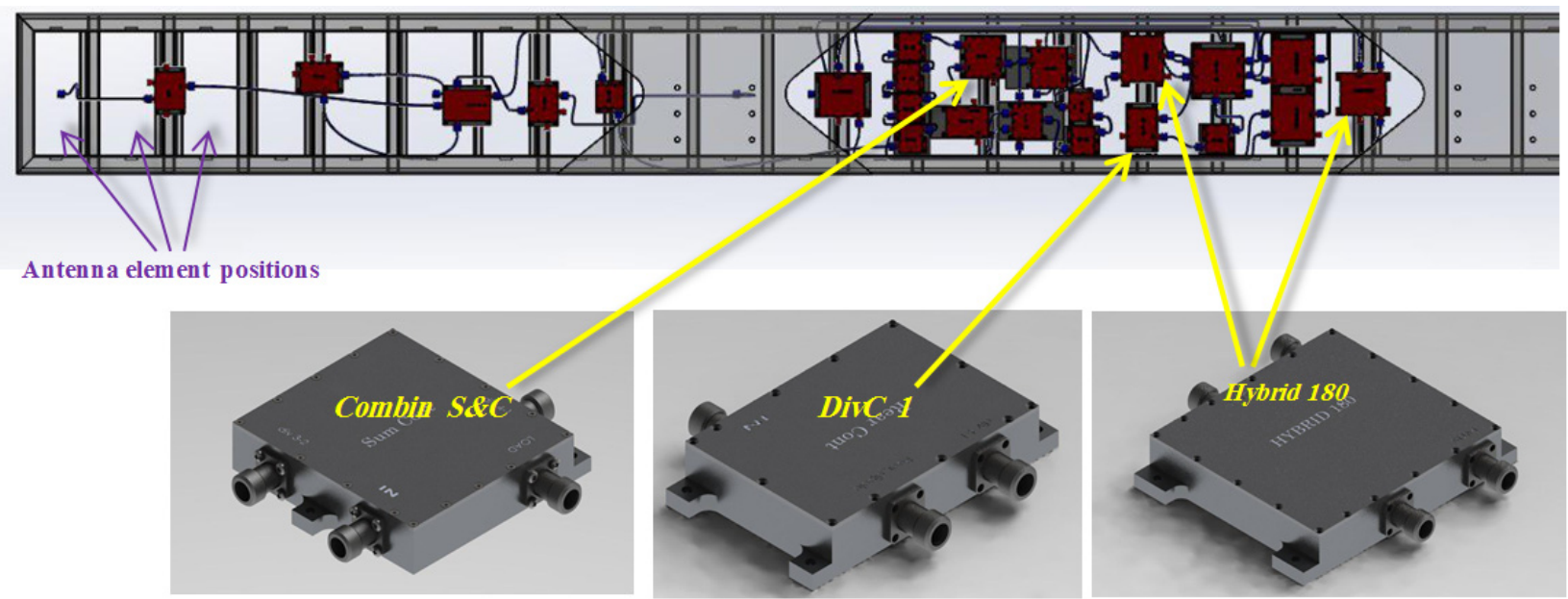

Fig. 8. Component placement and cabling of the feed network (enlarged picture of Combin_S\&C, DivC_1, and Hybrid180 respectively).

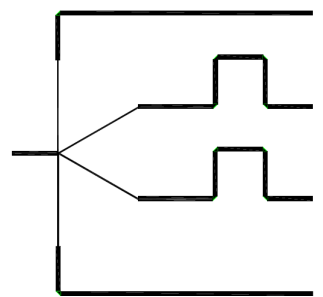

(a)

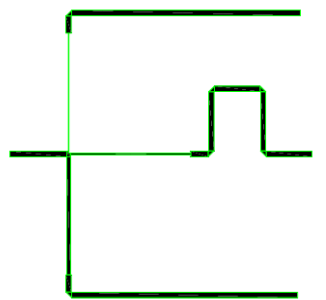

(b) (c)

Fig. 9. Layout configurations of a 1-to-4 divider (Div1-1 in Fig. 5), a 1-to-3 divider (Div3 in Fig. 5), and a 1-to-2 divider (DivD_2 in Fig. 5).

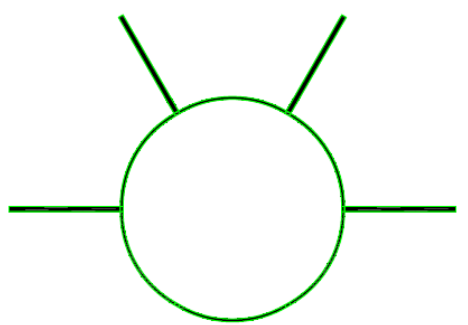

(a)

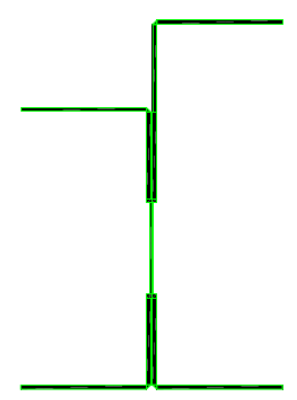

(b)

Fig. 10. Layout configurations of hybrid couplers (Hybrid 180 in Fig. 5), and directional coupler (Combin $\mathrm{S} \& \mathrm{C}$ in Fig. 5). for CTRL beam, namely Combin_S\&C, a simple single section coupled line coupler is designed and fabricated as shown in Fig. 10.

\section{Fabrication Results}

First, the complete feed network, which is a 6-meter long metallic structure (33 elements of $19 \mathrm{~cm}$ ), has been manufactured part by part, assembled in the metallic frame, and tested without dipole elements mounted. Due to length limitation, the antenna structure however was made for only 29 antenna elements. Therefore, the two end ports of the feed network at each side, four ports in total, were nullified, as shown in Fig. 11 while being tested outside Tehran. Figure 12 shows the measured pattern of 29 element array based on complete S-parameter measurement of feed network, meaning Sil to Si29 for all three beams SUM, DIFF and CTRL. The final $3 \mathrm{~dB}$ beam width of SUM is $4^{\circ}$ with $19 \mathrm{~dB}$ side lobe. There is a reasonable compliance between the expected simulation and measured results. The discrepancy is due to several factors mainly omission of two elements from the sides of the array, as well as the limited accuracy of cable length connecting feed network components besides none-ideal behavior of components.

Existing parasitic objects including real ground significantly contribute in open air measurements which are underway. Also the array has been tested in two cases, once with and once without rear dipole at the back. The results have shown that signal processing is able to remove the error caused by non-perfect CTRL beam especially at antennas back side.

Having the experience of design and fabrication of a metal framed 6-meter array for monopulse radar application, the most difficult problem in the design and fabrication is its complicated mechanics and assembly compared to few-piece solutions such as reflectors. However its flexibility, which comes from its modular design, worths spending on it. Yet, for smaller SSR arrays, printed solutions seem to be the best option. 


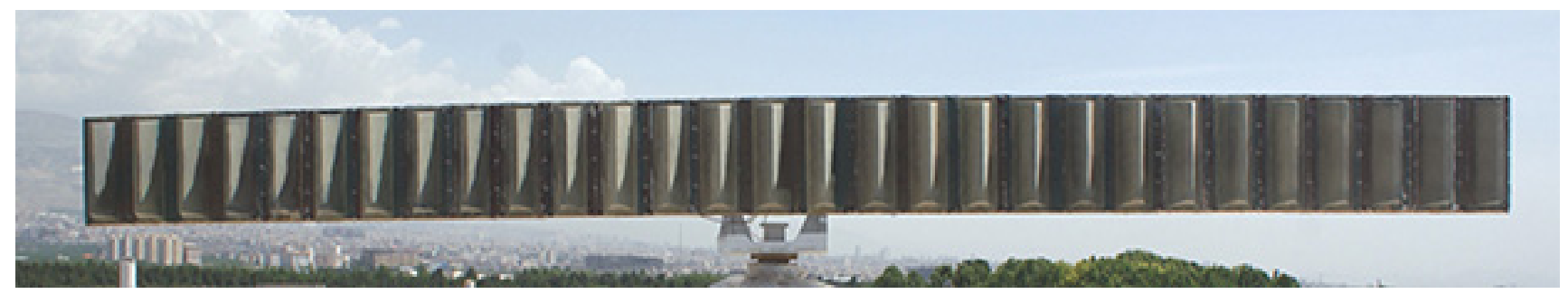

Fig. 11. Final array tested together with the rest of SSR system outside Tehran.

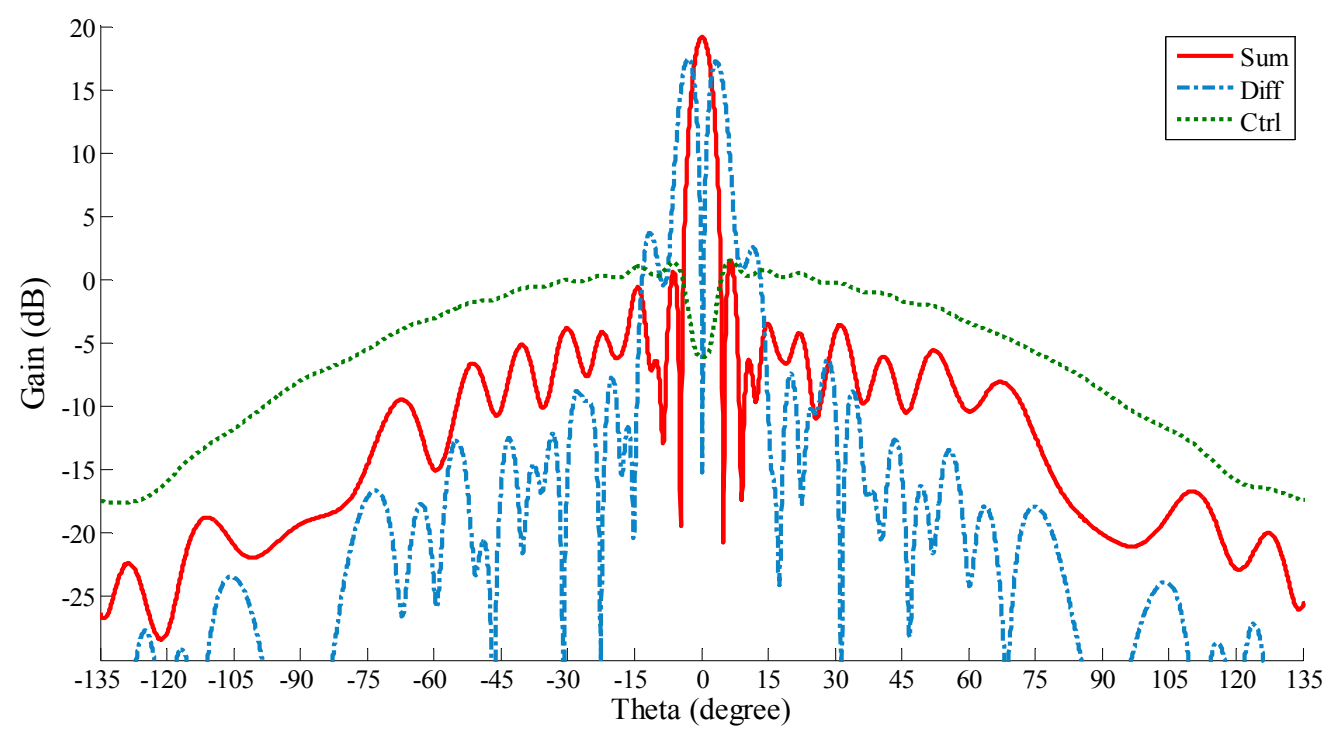

Fig. 12. Radiation pattern of beams based on feed network measurement in $1030 \mathrm{MHz}$.

\section{Conclusion}

In this paper, an efficient three beam antenna array for monopulse SSR applications was presented. The beam forming network provided three SUM, DIFF and CTRL beams, which are necessary for aircraft tracking and interrogating, out of one network. A new high gain dual-dipole antenna element was designed and fabricated for this array. A Taylor distribution was used for SUM and DIFF beams and a combination of SUM and center element of array formed the CTRL beam with a null in broadside direction. An optimized feed network was designed in order to establish all three beams with minimum RF component. The array has been set up successfully with 29 elements instead of 33 due to size limitations.

\section{References}

[1] STEVENS, M. C. New developments in secondary-surveillance radar. Electronics and Power, 1985, vol. 31, no. 6, p. 463-466. DOI: $10.1049 /$ ep.1985.0286

[2] STEVENS, M.C. Secondary Surveillance Radar. Boston and London: Artech House, 1988. ISBN-13: 978-0890062920

[3] INTERNATIONAL CIVIL AVIATION ORGANIZATION. Aeronautical telecommunications, annex 10, vol. 4. International Standards and Recommended Practices and Procedures for Air Navigation Services. $5^{\text {th }}$ ed., July 2014.
[4] WIRTH, W.D. Radar Techniques Using Array Antennas. $2^{\text {nd }}$ ed. IET, 2001. ISBN: 978-1-84919-698-7. DOI: 10.1049/PBRA026E

[5] KIM, S. G., CHANG, K. Low-cost monopulse antenna using bidirectionally-fed microstrip patch array. Electronics Letters, 2003, vol. 39 , no. 20 , p. 1428-1429. DOI: 10.1049/el:20030963

[6] ABDOLAHI, M., ASKARI, G., SADEGHI, H. M. A new seriesfed printed dipole array antenna for SSR. In 2017 Iranian Conference on Electrical Engineering (ICEE), Tehran (Iran), 2017, p. 1906-1910. DOI: 10.1109/IranianCEE.2017.7985366

[7] TEKKOUK, K., ETTORRE, M., LE COQ, L. et al. SIW pillbox antenna for monopulse radar applications. IEEE Transactions on Antennas and Propagation, 2015, vol. 63, no. 9, p. 3918-3927. DOI: 10.1109/TAP.2015.2446996

[8] ZHANG, H. Z., GRANET, C., SPREY, M. A. A compact Ku-band monopulse horn. Microwave and Optical Technology Letters, 2002, vol. 34, no. 1, p. 9-13. DOI: 10.1002/mop.10357

[9] ELLIOTT, R. D., CLARRICOATS, P. J. B. Corrugated waveguide monopulse feed. Electronics Letters, 1980, vol. 16, no. 9, p. 324-325. DOI: 10.1049/el:19800232

[10] REZAZADEH, N., SHAFAI, L. Ultrawideband monopulse antenna with application as a reflector feed. IET Microwaves, Antennas and Propagation, 2016, vol. 10, no. 4, p. 393-400. DOI: 10.1049/iet-map.2015.0437

[11] WANG, Y., DOU, W., BI, B. W-band axially displaced monopulse dual-reflector antenna for inter-satellite communications. IET Microwaves, Antennas and Propagation, 2016, vol. 10, no. 7, p. 742-747. DOI:10.1049/iet-map.2015.0559

[12] VESELY, J. History of radar and surveillance technology in the Czech Republic. In 18th International Radar Symposium (IRS). Prague (Czech Republic), 2017. DOI: 10.23919/IRS.2017.8008086 
[13] BEZOUSEK, P., CHYBA, M., SCHEJBAL, V., et al. Combined antenna array for primary and secondary surveillance radars. In 2014 IEEE-APS Topical Conference on Antennas and Propagation in Wireless Communications (APWC). Palm Beach (Netherlands Antilles), 2014. DOI: 10.1109/APWC.2014.6905572

[14] JACOVITTI, C. Performance analysis of monopulse receivers for secondary surveillance radar. IEEE Transactions on Aerospace and Electronic Systems, 1983, vol. 19, no. 6, p. 884-897. DOI: 10.1109/TAES.1983.309400

[15] SCHEJBAL, V., BEZOUSEK, P., PIDANIC, J., et al. Secondary surveillance radar antenna [Antenna Designer's Notebook]. IEEE Antennas and Propagation Magazine, 2013, vol. 55, no. 2, p. 164-170. DOI: 10.1109/MAP.2013.6578015

[16] NOORAEI YEGANEH, A., NAJMOLHODA, S. H., SEDIGHY, S. H., et al. New compact planar wideband antenna with flat gain and good pattern stability. Microwave and Optical Technology Letters, 2016, vol. 58, no. 11, p. 2548-2554. DOI: 10.1002/mop.30092
[17] TOHYA, K., URABE, M. Multibeam Radar System. U.S. Patent No. 6,137,434. 24 Oct. 2000.

[18] OLIVERI, G. Multibeam antenna arrays with common subarray layouts. IEEE Antennas and Wireless Propagation Letters, 2010, vol. 9, p. 1190-1193. DOI: 10.1109/LAWP.2010.2100073

[19] PEDERSEN, M. Ø., HANSEN, K., THOMSEN, A. K., et al. $\mathrm{X}$-band surveillance radar antenna with integrated IFF antenna. In IEEE Radar Conference (RADAR). Kansas City (MO, USA), 2011 p. 172-174. DOI: 10.1109/RADAR.2011.5960522

[20] BEZOUSEK, P., CHYBA, M., SCHEJBAL, V., et al. Dual frequency band integrated antenna array. In 7 th European Conference on Antennas and Propagation (EuCAP). Gothenburg, (Sweden), 2013, p. 2137-2141.

[21] ELLIOT, R. S. Antenna Theory and Design. Rev ed. John Wiley \& Sons, 2006. ISBN: 9788126508198

[22] POZAR, D. M. Microwave Engineering (Chapter: Transmission lines and waveguides, p. 143-149). 3 ed. Wiley, 2005. ISBN: 9780471644514 